Tôhoku Math. J.

41 (1989), 609-618

\title{
ASYMPTOTIC ALMOST PERIODIC SOLUTIONS FOR STOCHASTIC DIFFERENTIAL EQUATIONS
}

\author{
CONSTANTIN VÂRSAN
}

(Received June 23, 1988)

1. Introduction. We consider stochastic differential equations with asymptotic almost perodic coefficients and give sufficient conditions for a bounded solution to be asymptotically almost periodic in distribution. The case of almost periodic coefficients has been discussed in many articles and the total stability property is an important concept for the existence of an asymptotically almost periodic solution for ordinary and functional differential equations, see [1], [2] as references. A total stability concept is necessary also for stochastic differential equations, but we have to adopt an adequate strategy since the convergence in distribution of the initial conditions is too large for giving the convergence in $L_{2}(\Omega, P)$ of the corresponding solutions.

To avoid such a difficulty we do not fix the basic probability space $\{\Omega, \mathscr{F}, P\}$ and use Skorohod's theorem for defining equivalent stochastic differential equations which are more suitable for our purpose.

2. Formulation of the problem and the main result. Let $f_{i}: I \times R^{n} \rightarrow R^{n}, i=$ $0,1, \cdots, m$, be continuous and asymptotically almost periodic in the variable $t \in I$, $I=[0, \infty)$, uniformly with respect to $x$ belonging to compact sets in $R^{n}$; i.e. for any $\varepsilon>0$ and $K \subseteq R^{n}$ fixed there exist $l(\varepsilon, K)>0$ and $T(\varepsilon)>0$ such that any interval of length $l$ contains a $\tau$ fulfilling $\left|f_{i}(t+\tau, x)-f_{i}(t, x)\right|<\varepsilon$, for all $t \geqslant T, x \in K$. It is equivalent to the property that any sequence of real numbers $\left\{t_{k}\right\} \uparrow \infty$ contains a subsequence $\left\{t_{k}^{\prime}\right\} \uparrow \infty$ such that $\left\{f_{i}\left(t+t_{k}^{\prime}, x\right)\right\}_{k \geqslant 1}$ is convergent uniformly in $t \in I$ and $x$ in compact sets in $R^{n}$ (see [1], [2]).

Denote by $L^{+}\left(f_{0}, \cdots, f_{m}\right)$ the set of functions $g_{i}(t, x):[0, \infty) \times R^{n} \rightarrow R^{n}, \quad i=$ $0,1, \cdots, m$, which are obtained as limits of $f_{i}(t, x)$, i.e., $g_{i}(t, x)=\lim _{t_{k} \rightarrow \infty} f_{i}\left(t+t_{k}, x\right), i=$ $0,1, \cdots, m$, uniformly in $t \geqslant 0$ and $x$ in compact sets in $R^{n}$.

Let $w(t), t \geqslant 0$ be an $m$-dimensional Wiener process over the probability space $\{\Omega, \mathscr{F}, P\}$.

Let $\left\{\mathscr{F}_{t}\right\}_{t \geqslant 0}$ be an increasing family of $\sigma$-algebras in $\mathscr{F}$ such that the $\sigma$-algebra generated by $w(t+s)-w(t), s>0$, is independent of $\mathscr{F}_{t}$ for each $t \geqslant 0$. Let $x_{0}$ be $\mathscr{F}_{0}$-measurable and $x_{0} \in L_{p}(\Omega, P)$. The following stochastic differential equation

$$
d x=f_{0}(t, x) d t+\sum_{i=1}^{m} f_{i}(t, x) d w_{i}(t), \quad t \geqslant 0, \quad x(0)=x_{0}
$$


has a unique solution $x(t)$, on the interval $[0, \infty)$, if

$$
\begin{gathered}
\left|f_{i}(t, x)\right| \leqslant K(1+|x|), \quad\left|f_{i}\left(t, x_{2}\right)-f_{i}\left(t, x_{1}\right)\right| \leqslant K_{N}\left|x_{2}-x_{1}\right| \\
\text { for all } t \geqslant 0, \quad x \in R^{n}, \quad x_{1}, x_{2} \in R^{n}, \quad\left|x_{j}\right| \leqslant N .
\end{gathered}
$$

In addition, for each $p \geqslant 2$, we have $E|x(t)|^{p}<\infty$, for any $t \geqslant 0$, if $E\left|x_{0}\right|^{p}<\infty$.

The solutions for (1) are studied using limiting equations

$$
d x=g_{0}(t, x) d t+\sum_{i=1}^{m} g_{i}(t, x) d w_{i}(t), \quad t \geqslant 0
$$

where

$$
\left(g_{0}, \cdots, g_{m}\right) \in L^{+}\left(f_{0}, \cdots, f_{m}\right) .
$$

Remark 1. For our purpose we do not need to and it is suitable not to fix in advance the probability space $\{\Omega, \mathscr{F}, P\}$. The auxiliary probability space will be $\{\tilde{\Omega}, \tilde{\mathscr{F}}, \tilde{P}\}$ where $\tilde{\Omega}=\bar{\Omega} \times \Omega, \tilde{\mathscr{F}}=\overline{\mathscr{F}} \times \mathscr{F}, \tilde{P}=\bar{P} \times P$, and $\bar{\Omega}=[0,1), \overline{\mathscr{F}}$ is the $\sigma$-algebra of Borel sets in $[0,1)$, and $\bar{P}$ is the Lebesgue measure. The solutions in (1) and (2) are defined considering $w(t), t \geqslant 0$, a Wiener process on $\{\Omega, \mathscr{F}, P\}$ with the reference family of $\sigma$-algebras $\left\{\mathscr{F}_{t}\right\}_{t \geqslant 0}$ and initial condition $x(0)=x_{0}$ a random vector in $\{\bar{\Omega}, \overline{\mathscr{F}}\}$.

Denote by $\mathscr{P}$ the set of probability measures on $R^{n}$. Let $I_{1} \subseteq I \subset R$ be some intervals.

DeEFinItion 1 . We say that $P_{k}: I \rightarrow \mathscr{P}, k \geqslant 1$, is weakly compact uniformly with respect to $t \in I_{1}$ if there exists $\Pi: I \rightarrow \mathscr{P}$ and a subsequence $\left\{\boldsymbol{P}_{k^{\prime}}\right\} \subseteq\left\{\boldsymbol{P}_{\boldsymbol{k}}\right\}$ such that $\lim _{k^{\prime} \rightarrow \infty} \int_{R^{n}} \varphi(x) P_{k^{\prime}}(t, d x)=\int_{R^{n}} \varphi(x) \Pi(t, d x), t \in I$, uniformly with respect to $t \in I_{1}$, for any $\varphi \in C_{b}\left(R^{n}\right), \varphi$ Lipschitz continuous.

Let $x(t), t \geqslant 0$, be a solution in (1). Denote by $P(t, d x), t \geqslant 0$, the probability measure on $R^{n}$ generated by $x(t)$.

Definition 2. We say that a solution $x(t), t \geqslant 0$, in (1) is asymptotically almost periodic in distribution if $P_{k}(t, d x)=P\left(t+t_{k}, d x\right), k \geqslant 1$, is weakly compact uniformly in $t \in[0, \infty)$, for any sequence $\left\{t_{k}\right\} \subset[0, \infty), \lim t_{k}=\infty$.

Assume that (1) has a bounded solution in $L_{p}(\Omega, P), p>2$, i.e., there exists $\tilde{x}(t)$, $t \geqslant 0$, a solution for (1) such that

$$
\sup _{t \geqslant 0} E|\tilde{x}(t)|^{p} \leqslant M<\infty \quad \text { for some } \quad p>2,
$$

and we call it an $L_{p}$-bounded solution.

Denote by $\mathscr{H}$ the set of measurable processes $h:[0, \infty) \times \widetilde{\Omega} \rightarrow R^{n \times(m+1)}$ which are continuous in the variable $t \in[0, \infty)$ and for each $t>0, h(t, \cdot)$ is $\tilde{\mathscr{F}}_{t}$-adapted, $\tilde{\mathscr{F}}_{t}=$ $\overline{\mathscr{F}} \times \mathscr{F}{ }^{*}$

For each $s \geqslant 0$ we need to consider solutions of the following equation 


$$
\begin{aligned}
& d x=f_{0}(t+s, x) d t+\sum_{i=1}^{m} f_{i}(t+s, x) d w_{i}(t), \quad t \geqslant 0, \\
& x(0)=x_{0}(\bar{\omega}),
\end{aligned}
$$

where $x_{0} \in L_{2}(\bar{\Omega} ; \bar{P})$ and $w(t), t \geqslant 0$, is the fixed Wiener process on the probability space $\{\Omega, \mathscr{F}, P\}$ with the given reference family of $\sigma$-algebras $\left\{\mathscr{F}_{t}\right\}_{t \geqslant 0} \subseteq \mathscr{F}$. The equation $\left(1_{s}\right)$ is considered on the new space $\{\tilde{\Omega}, \tilde{\mathscr{F}}, \tilde{P}\}$ and the corresponding reference family given by $\tilde{\mathscr{F}}_{t}=\overline{\mathscr{F}} \times \mathscr{F}_{t}$.

In a similar way, for each $\left(g_{0}, \cdots, g_{m}\right) \in L^{+}\left(f_{0}, \cdots, f_{m}\right)$ and $s \geqslant 0$ we consider the following equation

$$
\begin{aligned}
& d x=g_{0}(t+s, x) d t+\sum_{i=1}^{m} g_{i}(t+s, x) d w_{i}(t), \\
& x(0)=x_{0}(\bar{\omega}) .
\end{aligned}
$$

Any solution for $\left(1_{s}\right)$ and $\left(2_{s}\right)$ is a measurable process $x(t, \tilde{\omega}):[0, \infty) \times \tilde{\Omega} \rightarrow R^{n}$, whose trajectories are continuous functions a.e. $(\widetilde{P})$ and with $x(t, \cdot) \tilde{\mathscr{F}}_{t}$-adapted. Denote $f=\left(f_{0}, \cdots, f_{m}\right), g=\left(g_{0}, \cdots, g_{m}\right)$ and define $f_{s}(t, x)=\left(f_{0}(t+s, x), \cdots, f_{m}(t+s, x)\right), g_{s}(t, x)=$ $\left(g_{0}(t+s, x), \cdots, g_{m}(t+s, x)\right)$. The solutions for $\left(1_{s}\right)$ and $\left(2_{s}\right)$ are denoted by $x\left(t, f_{s}, x_{0}\right)$ and $x\left(t, g_{s}, x_{0}\right)$, respectively.

DEFINITION 3. We say that $x\left(t, f_{s}, x_{0}\right)$ in $\left(1_{s}\right)$ is totally stable if for any $\varepsilon>0$ there exists $\delta(\varepsilon)>0$ such that $\sup _{t \geqslant 0} \tilde{E}\left|x\left(t, f_{s}+h, x_{0}+v_{0}\right)-x\left(t, f_{s}, x_{0}\right)\right|^{2} \leqslant \varepsilon$, for any $v_{0} \in$ $L_{2}(\bar{\Omega}, \bar{P})$ and $h \in \mathscr{H}$ fulfilling $\bar{E}\left|v_{0}\right|^{2} \leqslant \delta(\varepsilon)$ and $\sup _{t \geqslant 0} \tilde{E}|h(t, \tilde{\omega})|^{2} \leqslant \delta(\varepsilon)$.

An $L_{p}$-bounded solution for $\left(1_{s}\right)$ is defined by $\sup _{t \geqslant 0} \tilde{E}\left|x\left(t, f_{s}, x_{0}\right)\right|^{p} \leqslant M<\infty$ and similarly for $(2)$.

Definition 4. We say that the equation (1) (or (2)) is totally stable if any $L_{p}$-bounded solution for $\left(1_{s}\right)$ (or $\left(2_{s}\right)$ ) is totally stable for any $s \geqslant 0$.

THEOREM 1. Assume $\left(\mathrm{i}_{1}\right)$ and $\left(\mathrm{i}_{2}\right)$ are fulfilled and $(1)$ is totally stable. Then any $L_{p}$-bounded solution $x(t)$ for $(1)$ is asymptotically almost periodic in distribution.

THEOREM 2. Assume $\left(\mathrm{i}_{1}\right)$ and $\left(\mathrm{i}_{2}\right)$ are fulfilled. Let $\left(g_{0}, \cdots, g_{m}\right) \in L^{+}\left(f_{0}, \cdots, f_{m}\right)$ and the corresponding equation (2) is totally stable. Then any $L_{p}$-bounded solution $\bar{x}(t)$ in (1) is asymptotically almost periodic in distribution.

REMARK 2. From Theorem 1 or 2 follows that for any $\varphi \in C_{b}\left(R^{n}\right), \varphi$ Lipschitz continuous, the real function $f(t)=E \varphi\left(\tilde{x}\left(t, x_{0}\right)\right)$, defined on $I=[0, \infty)$, is an asymptotic almost periodic function and the results in [1] associated to it apply to this case in dependence of the function $\varphi$ we choose. More generally, the space of Lipschitz continuous functions in $C_{b}\left(R^{n}\right)$ is separable and there is a metric $\rho$ on $\mathscr{P}$ such that any $P(t, d x): I \rightarrow(\mathscr{P}, \rho)$ which corresponds to an asymptotically almost periodic solution with $p \geqslant 4$ has an almost periodic components $\Pi: R \rightarrow \mathscr{P}$, such that $\lim _{t \rightarrow \infty} \rho(P(t), \Pi(t))=0$ 
and the corresponding decomposition $P(t, d x)=\Pi(t, d x)+Q(t, d x)$ is unique, where $Q(t, d x)$ is a finite Random measure.

3. Some auxiliary results and proof of the theorems. In order to get uniform convergence of a sequence $P_{k}:[0, \infty) \rightarrow \mathscr{P}$, with respect to $t \geqslant 0$, where $P_{k}(t, d x)$ represents the marginal distribution of a fixed solution for (1) or (2), we need to work with strong solutions and their continuous dependence with respect to perturbations. Denote by $\{\bar{\Omega}, \overline{\mathscr{F}}, \bar{P}\}$ a new probability space, where $\bar{\Omega}=[0,1), \bar{P}$ the Lebesgue measure and $\overline{\mathscr{F}}$ the $\sigma$-algebra generated by the intervals $[a, b), 0 \leqslant a<b<1$. If we take a solution $x(t)$, $t \geqslant 0$, for (1) and $\left\{t_{k}\right\} \uparrow \infty$, then $x_{k}(t)=x\left(t+t_{k}, x_{0}\right), k \geqslant 1$, fulfils the following equation

$$
d x=f_{0}\left(t+t_{k}, x\right) d t+\sum_{i=1}^{m} f_{i}\left(t+t_{k}, x\right) d w_{i}^{k}(t),
$$

where $w_{i}^{k}(t)=w_{i}\left(t+t_{k}\right), i=1, \cdots, m$, is a new Wiener process on $\{\Omega, \mathscr{F}, P\}$. The solution $x_{k}(t)$ and the corresponding equation (3) is well defined for any $t \in[a, \infty)$, where $a \leqslant 0$ is fixed arbitrarily, if $k$ is sufficiently large such that $a+t_{k} \geqslant 0$.

Moreover, the probability $P_{k}(t, d x), t \in[a, \infty)$, generated by $x_{k}(t)$ on $R^{n}$, coincides with the probability generated by the solution $x_{k}(t), t \in[a, \infty)$ of the following equation

$$
\begin{aligned}
& d x=f_{0}\left(t+t_{k}, x\right)+\sum_{i=1}^{m} f_{i}\left(t+t_{k}, x\right) d w_{i}(t), \quad t \in[a, \infty), \\
& x(a)=\bar{x}_{k}(\bar{\omega}),
\end{aligned}
$$

where $w_{i}(t), t \in[a, \infty)$ is a fixed Wiener process on $\{\Omega, \mathscr{F}, P\}, \bar{x}_{k}$ is a random vector on $\{\bar{\Omega}, \overline{\mathscr{F}}, \bar{P}\}$ with the distribution $P_{k}(a, d x)$ on $R^{n}$.

In addition, if $P_{k}(a, d x)$ converges weakly to $P_{0}(d x)$, then there exists a random vector $\bar{x}_{0}(\bar{\omega})$ on $\{\bar{\Omega}, \overline{\mathscr{F}}, \bar{P}\}$ such that the distribution of $\bar{x}_{0}$ is $P_{0}, \lim _{k \rightarrow \infty} \bar{x}_{k}(\bar{\omega})=\bar{x}_{0}(\bar{\omega})$ a.e. with respect to $\bar{P}$; it is ensured by Skorohod's theorem (see [4]).

Assuming that $\left(i_{1}\right)$ and $\left(i_{2}\right)$ are fulfilled for $(1)$ and then applying the above remark to the $L_{p}$-bounded solution $\tilde{x}(t), t \geqslant 0$, one may get that $\tilde{x}_{k}(t)=\tilde{x}\left(t+t_{k}\right), t \geqslant a, k \geqslant k_{0}$, fulfils

$$
\sup _{t \geqslant a} E\left|\tilde{x}_{k}(t)\right|^{p} \leqslant M<\infty \quad \text { for some } \quad p>2
$$

and therefore the corresponding distributions $\left\{P_{k}(t, d x)\right\}_{k \geqslant k_{0}}$ are weakly compact for any $t \geqslant a$ (see [4]). In addition, denoting by $\left\{\widetilde{P}_{k}(a, d x)\right\}_{k \geqslant 1}$ a weakly convergent subsequence of $\left\{P_{k}(a, d x)\right\}_{k \geqslant k_{0}}$ and $P_{0}$ its limit, by Skorohod's theorem we get $\tilde{x}_{k}, \tilde{x}_{0}$ random vectors on $\{\bar{\Omega}, \overline{\mathscr{F}}, \bar{P}\}$ such that

$$
\bar{E}\left|\tilde{x}_{k}\right|^{p} \leq M, \quad \bar{E}\left|\tilde{x}_{0}\right|^{p} \leq M, \quad \lim _{k \rightarrow \infty} \tilde{x}_{k}(\bar{\omega})=\tilde{x}_{0}(\bar{\omega}), \text { a.e. } \bar{P},
$$

where $\bar{E}$ means the expectation with respect to $\bar{P}$, and the constants $M$ and $p$ in (6) are the same with those appearing in (5). 
Using (6) we get the uniform integrability of the sequence $\left|\tilde{x}_{k}(\bar{\omega})-\tilde{x}_{0}(\bar{\omega})\right|^{2}, k \geqslant 1$, on $\{\bar{\Omega}, \overline{\mathscr{F}}, \bar{P}\}$ and the convergence of $\tilde{x}_{k}$ to $\tilde{x}_{0}$ in $L_{2}(\bar{\Omega}, \bar{P})$

$$
\lim _{k \rightarrow \infty} \bar{E}\left|\tilde{x}_{k}-\tilde{x}_{0}\right|^{2}=0 \text {. }
$$

LEMma 1. Let the conditions $\left(\mathrm{i}_{1}\right),\left(\mathrm{i}_{2}\right)$ and $p \geqslant 4$ be fulfilled for (1), and $\tilde{P}(t, d x)$, $t \geqslant 0$, be the corresponding probability generated by $\tilde{x}(t)$. Then for any $a<0$ and $\left\{t_{k}\right\} \uparrow \infty$ there exists a subsequence $\left\{t_{k^{\prime}}\right\} \uparrow \infty$ and $\tilde{P}(t, d x) \in \mathscr{P}$ such that $\widetilde{P}\left(t+t_{k^{\prime}}, d x\right)=P_{k^{\prime}}(t, d x)$ is weakly convergent to $\widetilde{P}(t, d x)$ for any $a \leqslant t<\infty$.

Proof. Denote $\tilde{x}(t)=\tilde{x}\left(t+t_{k}\right)$ and for $k$ sufficiently large, $k \geqslant k_{0}, \tilde{x}_{k}(t)$ is defined by (3) for any $t \geqslant a$, with $\tilde{x}_{k}(a)=\tilde{x}\left(a+t_{k}\right)$.

By hypotheses

$$
E\left|x_{k}(a)\right|^{p} \leqslant M \quad \text { for some } p \geqslant 4 \text { for all } k \geqslant k_{0}
$$

and

$$
E\left|\tilde{x}_{k}\left(t^{\prime \prime}\right)-\tilde{x}_{k}\left(t^{\prime}\right)\right|^{4} \leqslant K(T)\left|t^{\prime \prime}-t^{\prime}\right|^{2} \quad \text { for all } t^{\prime}, t^{\prime \prime} \in[a, t], \quad k \geqslant k_{0} .
$$

Using Theorem 4.3 in $\left[4\right.$, p. 18] we get a sequence $\left\{\Pi_{k^{\prime}}\right\}$ of probabilities on $C\left([a, \infty) ; R^{n}\right)$ and $\Pi$ such that $\Pi_{k^{\prime}} \rightarrow \Pi$ weakly and the conclusion follows by restriction to $C\left([a, t] ; R^{n}\right)$.

Remark 3. Using Skorohod's theorem, the function $\widetilde{P}(t, d x) t \geqslant a$ in Lemma 1 can be generated for each $t$ as the marginal distribution of a continuous process and therefore $\widetilde{P}(t, d x)$ is weakly continuous in the variable $t$.

In addition, one may obtain that $\int_{R^{n}} \varphi(x) \widetilde{P}_{k^{\prime}}(t, d x)$ converges to $\int_{R^{n}} \varphi(x) \widetilde{P}(t, d x)$ uniformly in $t \in[a, T]$ for any $T>0$ and $\varphi$ Lipschitz continuous, $\varphi \in C_{b}\left(R^{n}\right)$, but it does not mean that $\widetilde{P}_{k^{\prime}}(t, d x)$ converges uniformly with respect to $t \in[0, \infty)$.

The next step is to prove that total stability of (1) or (2) implies the uniform convergence of $P_{k^{\prime}}(t, d x)$ with respect to $t \geqslant 0$.

LEMMA 2. Let the conditions $\left(\mathrm{i}_{1}\right)$ and $\left(\mathrm{i}_{2}\right)$ be fulfilled, and $\tilde{x}(t), t \geqslant 0$, be the $L_{p}$-bounded solution given by the hypothesis $\left(\mathrm{i}_{2}\right)$. Assume that there exists $g=\left(g_{0}, \cdots, g_{m}\right) \in L^{+}\left(f_{0}, \cdots, f_{m}\right)$ such that $(2)$ is totally stable. Then there exists a sequence $\left\{t_{k}\right\}_{k \geqslant 1} \subset[0, \infty), \lim t_{k}=\infty$, such that $\tilde{P}_{k}(t, d x)=\widetilde{P}\left(t+t_{k}, d x\right)$ is weakly convergent to a $P(t, d x)$ uniformly in $t \in[0, \infty)$. In addition, $P(t, d x)$ is generated by an $L_{p}$-bounded solution $v(t)$ for (2) fulfilling $\sup _{t \geqslant 0} \tilde{E}|v(t, \tilde{\omega})|^{p} \leqslant M<\infty$, and $\tilde{P}_{k}(t, d x)$ is generated by an $L_{p^{-}}$ bounded solution $v_{k}(t)$ in $\left(1_{t_{k}}\right)$ such that $\lim _{k \rightarrow \infty} \tilde{E}\left|v_{k}(t, \tilde{\omega})-v(t, \tilde{\omega})\right|^{2}=0$ uniformly in $t \geqslant 0$.

Proof. By hypotheses there exists $\left\{t_{k}\right\} \uparrow \infty$ such that $g_{i}(t, x)=\lim _{k \rightarrow \infty} f_{i}\left(t+t_{k}, x\right)$ uniformly in $t \geqslant 0$ and for $x$ in compact sets in $R^{n}, i=0,1, \cdots, m$.

By definition $\tilde{x}_{k}(t)=\tilde{x}\left(t+t_{k}, x_{0}\right), t \geqslant 0, k=1,2, \cdots$, is the solution in (3) with initial 
condition $\tilde{x}_{k}(0)=\tilde{x}\left(t_{k}\right)$ and using $\left(i_{2}\right)$ we get that $\left\{\tilde{P}\left(t_{k}, d x\right)\right\}_{k \geqslant 1}$ is tight and therefore weakly compact. Substract a subsequence if necessary and one obtains $\tilde{P}(0, d x) \in \mathscr{P}$ and $\widetilde{P}\left(t_{k}, d x\right)$ converges to $\widetilde{P}(0, d x)$ weakly. Using Skorohod's theorem we get $\tilde{x}_{k}(\bar{\omega}), \tilde{x}_{0}(\bar{\omega})$ random vectors on $\{\bar{\Omega}, \overline{\mathscr{F}}, \bar{P}\}$ such that the probabilities generated on $R^{n}$ by $\tilde{x}_{k}$ and $\tilde{x}_{0}$ coincide with $\widetilde{P}\left(t_{k}, d x\right)$ and $\widetilde{P}(0, d x)$ respectively, and (7) is fulfilled. Moreover the probability generated on $C\left([0, \infty) ; R^{n}\right)$ by $\tilde{x}_{k}(t), t \geqslant 0$, coincides with the probability generated on $C\left([0, \infty) ; R^{n}\right)$ by the solution $v_{k}(t, \bar{\omega})$ in (4) with initial condition $v_{k}(0)=\tilde{x}_{k}(\bar{\omega}), k \geqslant 1$. Take initial condition $v(0)=\tilde{x}_{0}(\bar{\omega})$ and denote $v(t, \tilde{\omega}), t \geqslant 0$, the corresponding solution for (2) where $g_{0}, \cdots, g_{m}$ are given by hypotheses. We shall show that

$$
\lim _{k \rightarrow \infty} \tilde{E}\left|v_{k}(t, \tilde{\omega})-v(t, \tilde{\omega})\right|^{2}=0 \quad \text { uniformly in } \quad t \in[0, \infty),
$$

where $\tilde{E}$ means the expectation with respect to the product measure $\tilde{P}=\bar{P} \times P$ on $(\bar{\Omega} \times \Omega, \overline{\mathscr{F}} \times \mathscr{F})=(\tilde{\Omega}, \tilde{\mathscr{F}})$. It is easily seen that $v_{k}(t, \tilde{\omega}), t \geqslant 0$, fulfils the following equation

$$
\begin{aligned}
d x= & g_{0}(t, x) d t+\sum_{i=1}^{m} g_{i}(t, x) d w_{i}(t)+\left[f_{0}\left(t+t_{k}, v_{k}(t)\right)-g_{0}\left(t, v_{k}(t)\right)\right] d t \\
& +\sum_{i=1}^{m}\left[f_{i}\left(t+t_{k}, v_{k}(t)\right)-g_{i}\left(t, v_{k}(t)\right)\right] d w_{i}(t), \\
x(0)= & \tilde{x}_{k}(\bar{\omega}) .
\end{aligned}
$$

On the other hand $v(t), t \geqslant 0$, fulfils

$$
\begin{aligned}
& d x=g_{0}(t, x) d t+\sum_{i=1}^{m} g_{i}(t, x) d w_{i}(t), \\
& x(0)=\tilde{x}_{0}(\bar{\omega}) .
\end{aligned}
$$

Denote $h_{i}^{k}(t, \tilde{\omega})=f_{i}\left(t+t_{k}, v_{k}(t, \tilde{\omega})\right)-g_{i}\left(t, v_{k}(t, \tilde{\omega})\right), i=0,1, \cdots, m$, where $\tilde{\omega}=(\bar{\omega}, \omega) \epsilon$ $\bar{\Omega} \times \Omega$. If we show that

$$
\lim _{k \rightarrow \infty} \tilde{E}\left|h_{i}^{k}(t, \tilde{\omega})\right|^{2}=0 \quad \text { uniformly in } \quad t \geqslant 0,
$$

then using (7) and total stability property of (12) we get

$$
\lim _{k \rightarrow \infty} \sup _{t \geqslant 0} \tilde{E}\left|v_{k}(t, \tilde{\omega})-v(t, \tilde{\omega})\right|^{2}=0,
$$

which implies the convergence conclusion in the lemma.

In order to prove (13) we notice that $\tilde{E}\left|v_{k}(t, \tilde{\omega})\right|^{p}=E\left|\tilde{x}\left(t+t_{k}\right)\right|^{p}$ and using $\left(\mathrm{i}_{2}\right)$ we have 


$$
\sup _{t \geqslant 0} \tilde{E}\left|v_{k}(t, \tilde{\omega})\right|^{p} \leq M<\infty \quad \text { for some } \quad p>2 .
$$

From (14) and (15) we get the $L_{p}$-boundedness of $v(t, \tilde{\omega}), t \geqslant 0$. Using (15) it is easily seen that for any $\delta \in(0,1)$ there is $N_{\delta}>0$ such that

$$
\tilde{P}\left\{\tilde{\omega}:\left|v_{k}(t, \tilde{\omega})\right|>N_{\delta}\right\} \leq \delta \quad \text { for all } t \geqslant 0, \quad k=1,2, \cdots .
$$

On the compact set $S_{\delta}=\left\{x \in R^{n}:|x| \leq N_{\delta}\right\}$ we have $\lim _{t_{k} \rightarrow \infty} f_{i}\left(t+t_{k}, x\right)=g_{i}(t, x)$, uniformly in $(t, x) \in I \times S_{\delta}, I=[0, \infty)$. Since $h_{i}^{k}(t, x)=f_{i}\left(t+t_{k}, x\right)-g_{i}(t, x)$ fulfils

$$
\left|h_{i}^{k}(t, x)\right| \leqslant \tilde{K}(1+|x|) \quad \text { for all } k \geqslant 1, \quad t \geqslant 0, \quad x \in R^{n},
$$

we get

$$
\left|h_{i}^{k}(t, \tilde{\omega})\right|^{2} \leqslant \bar{K}\left(1+\left|v_{k}(t, \tilde{\omega})\right|^{2}\right) \quad \text { for some constant } \quad \bar{K}>0 .
$$

By definition we have

$$
\tilde{E}\left|h_{i}^{k}(t, \tilde{\omega})\right|^{2}=\int_{A_{\delta}}\left|h_{i}^{k}(t, \tilde{\omega})\right|^{2} \tilde{P}(d \tilde{\omega}) \int_{\tilde{\Omega} \backslash A_{\delta}}\left|h_{i}^{k}(t, \tilde{\omega})\right|^{2} \tilde{P}(d \tilde{\omega}),
$$

where $A_{\delta}=\left\{\tilde{\omega} \in \tilde{\Omega}:\left|v_{k}(t, \tilde{\omega})\right|>N_{\delta}\right\}$.

Using (15), (16) and (18) in (19) one obtains that the first term on the right hand side of (19) can be made smaller than any positive number uniformly with respect to $t \geqslant 0, k=1,2, \cdots$, if we choose $\delta$ sufficiently small. On the other hand the second term on the right hand side of (19) converges to zero for $k \rightarrow \infty$ and $\delta>0$ fixed.

It allows one to conclude that (13) is fulfilled and the proof is complete.

Denote by $P^{x}$ the probability on $R^{n}$ generated by a random vector $x(\cdot)$.

LEMMA 3. Assume that (1) has an $L_{p}$-bounded solution $\hat{x}(t), t \geqslant 0$, and (1) is totally stable. Then for any $\left\{t_{k}\right\} \uparrow \infty$ there exist $\left\{s_{j}\right\} \subseteq\left\{t_{k}\right\},\left\{s_{j}\right\} \uparrow \infty$, and $x_{j}(t)$, solution for $\left(1_{s_{j}}\right)$, and $x(t) \in L_{2}(\tilde{\Omega}, \tilde{P})$ such that $\lim _{j \rightarrow \infty} \sup _{t \geqslant 0} \tilde{E}\left|x_{j}(t, \tilde{\omega})-x(t, \tilde{\omega})\right|^{2}=0$ and $P^{x_{j}(t)}=P^{\hat{x}\left(t+s_{j}\right)}$ for any $t \geqslant 0, j \geqslant 1$.

Proof. By definition, $\hat{x}\left(t+t_{k}\right)=\hat{x}_{k}(t)$ fulfils (3) with $x_{k}(0)=\hat{x}\left(t_{k}\right)$ and it generates a probability $\hat{\Pi}_{k}$ on $C\left([0, \infty) ; R^{n}\right)$ which coincides with $\Pi_{k}$ on $C\left([0, \infty) ; R^{n}\right)$ generated by the solution $x_{k}(t, \tilde{\omega})$ in $\left(1_{t_{k}}\right)$ with initial condition $x(0)=x_{k}(\bar{\omega})$. In addition

$$
\sup _{t \geqslant 0} \tilde{E}\left|x_{k}(t, \tilde{\omega})\right|^{p} \leqslant M<\infty \quad \text { for all } k \geqslant 1 .
$$

Using the above boundedness condition, we get a subsequence $\left\{x_{j}(\bar{\omega})\right\}_{j \geqslant 1} \subset$ $\left\{x_{k}(\bar{\omega})\right\}_{k \geqslant 1}$ corresponding to $\left\{s_{j}^{\prime}\right\} \subseteq\left\{t_{k}\right\},\left\{s_{j}^{\prime}\right\} \uparrow \infty$, which converges in $L_{2}(\bar{\Omega}, \bar{P})$ to $x_{0}(\bar{\omega})$ (see (6), (7)). On the other hand, there is a subsequence $\left\{s_{j}\right\} \subseteq\left\{s_{j}^{\prime}\right\},\left\{s_{j}\right\} \uparrow \infty$, such that $\left\{f\left(t+s_{j}, x\right)\right\}_{j \geqslant 1}$ converges uniformly in $t \geqslant 0$ and $x$ in compact sets in $R^{n}$.

Denote by $x_{j}(t, \tilde{\omega}), j \geqslant 1$, the corresponding solution in $\left(1_{s_{j}}\right)$ with initial condition 
$x(0)=x_{j}(\bar{\omega})$. We note that $x_{j}(t, \tilde{\omega})$ is a solution for the following equation

$$
\begin{aligned}
d x= & f_{0}\left(t+s_{l}, x\right) d t+\sum_{i=1}^{m} f_{i}\left(t+s_{l}, x\right) d w_{i}(t)+\left[f_{0}\left(t+s_{j}, x_{j}(t)\right)-f_{0}\left(t+s_{l}, x_{j}(t)\right)\right] d t \\
& +\sum_{i=1}^{m}\left[f_{i}\left(t+s_{j}, x_{j}(t)\right)-f_{i}\left(t+s_{l}, x_{j}(t)\right) d w_{i}(t),\right. \\
x(0)= & x_{j}(\bar{\omega}) .
\end{aligned}
$$

Using arguments similar to those in the proof of Lemma 2 (see (16)-(19)) we can prove that for any $\eta>0$ there is $l=l(\eta)$ such that we have

$$
\sup _{t \geqslant 0} \tilde{E}\left|h_{i}^{j}(t, \tilde{\omega})\right|^{2} \leq \eta, \quad \bar{E}\left|x_{j}(\bar{\omega})-x_{l}(\bar{\omega})\right|^{2} \leq \eta \quad \text { for any } \quad j \geqslant l(\eta),
$$

where $h_{i}^{j}(t, \tilde{\omega})=f_{i}\left(t+s_{j}, x_{j}(t)\right)-f_{i}\left(t+s_{l}, x_{j}(t)\right)$.

Using total stability property of $(1)$ we obtain that $\left\{x_{j}(t, \tilde{\omega})\right\}_{j \geqslant 1}$ is a Cauchy sequence in $L_{2}(\tilde{\Omega}, \widetilde{P})$, uniformly in $t \geqslant 0$, which ensures the convergence conclusion and the proof is complete.

Now we are in a position to prove the theorems.

Proof of Theorem 2. Let $\left(g_{0}, \cdots, g_{m}\right) \in L^{+}\left(f_{0}, \cdots, f_{m}\right)$. By hypotheses, Lemma 2 applies and the corresponding equation (2) is totally stable. Let $\left\{t_{k}\right\} \uparrow \infty$ be a sequence such that $\tilde{P}\left(t+t_{k}, d x\right)=\tilde{P}_{k}(t, d x)$ converges weakly to $P(t, d x)$, uniformly in $t \geqslant 0$.

Take an arbitrary sequence $\left\{h_{k}^{\prime}\right\} \uparrow \infty$. Then there exists a subsequence $\left\{h_{k}\right\} \subseteq\left\{h_{k}^{\prime}\right\}$, $\left\{h_{k}\right\} \uparrow \infty$ such that $h_{k}=t_{k}+s_{k}, h_{k}>2 t_{k}$.

We have $\left\{s_{k}\right\} \uparrow \infty$ and $P\left(t+s_{k}+t_{k}, d x\right)$ converges weakly to $P\left(t+s_{k}, d x\right)$ for $t_{k} \rightarrow \infty$ uniformly with respect to $t \geqslant 0$ and $\left\{s_{k}\right\}$. Using again Lemma 2 we know that $P(t, d x), t \geqslant 0$ is generated by a solution $v(t)$ in (2) (see (12)) which fulfils the $L_{p}$-boundedness condition $\left(i_{2}\right)$. Also the equation (2) fulfils $\left(i_{1}\right)$ and in addition is totally stable.

We notice that $v_{k}(t)=v\left(t+s_{k}\right), t \geqslant 0$ is a solution for the following equation

$$
\begin{aligned}
& d x=g_{0}\left(t+s_{k}, x\right) d t+\sum_{i=1}^{m} g_{i}\left(t+s_{k}, x\right) d w_{i}^{k}(t), \\
& x(0)=v\left(s_{k}, \tilde{\omega}\right),
\end{aligned}
$$

where $w_{i}^{k}(t)=w_{i}\left(t+s_{k}\right), t \geqslant 0$, is a new Wiener process on $\{\Omega, \mathscr{F}, P\}$ with the reference family of $\sigma$-algebras $\left\{\mathscr{F}_{t}^{k}\right\}, t \geqslant 0, \mathscr{F}_{t}^{k}=\mathscr{F}_{t+s_{k}}$.

The same procedure as in Lemma 2 applies here and (20) is replaced by an equivalent one as follows.

Denote by $P_{k}(t)$ the probability generated on $R^{n}$ by $v_{k}(t)$. The same probabilities $P_{k}(t), t \geqslant 0$, will be generated by the solution of the following equation 


$$
\begin{aligned}
& d x=g_{0}\left(t+s_{k}, x\right) d t+\sum_{i=1}^{m} g_{i}\left(t+s_{k}, x\right) d w_{i}(t), \quad t \geqslant 0, \\
& x(0)=x_{k}(\bar{\omega}),
\end{aligned}
$$

where $w(t)$ is the fixed Wiener process on probability space $\{\Omega, \mathscr{F}, P\}$ with the reference family $\left\{\mathscr{F}_{t}\right\}, t \geqslant 0$, and $x_{k}$ is a random vector on $\{\bar{\Omega}, \overline{\mathscr{F}}, \bar{P}\}$ with the distribution $P_{k}(0)$.

The new probability space will be $\{\tilde{\Omega}, \tilde{\mathscr{F}}, \widetilde{P}\}$ and $\tilde{\mathscr{F}}_{t}=\mathscr{F}_{t} \times \overline{\mathscr{F}}, t \geqslant 0$, is the associated reference family of $\sigma$-algebras (see Remark 1 ).

In the sequel the solution in (21) will be denoted again by $v_{k}(t)$ and it is easily seen that $v_{k}(t), k \geqslant 1, t \geqslant 0$, fulfils the following equation

$$
\begin{aligned}
d x= & g_{0}\left(t+s_{l}, x\right) d t+\sum_{i=1}^{m} g_{i}\left(t+s_{l}, x\right) d w_{i}(t)+\left[g_{0}\left(t+s_{k}, v_{k}(t)\right)-g_{0}\left(t+s_{l}, v_{k}(t)\right)\right] d t \\
& +\sum_{i=1}^{m}\left[g_{i}\left(t+s_{k}, v_{k}(t)\right)-g_{i}\left(t+s_{l}, v_{k}(t)\right)\right] d w_{i}(t) .
\end{aligned}
$$

Arguments similar to those in Lemma 2 apply and using total stability of (2) we get $\left\{\tilde{s}_{k}\right\} \subseteq\left\{s_{k}\right\},\left\{\tilde{s}_{k}\right\} \uparrow \infty$ such that $\tilde{v}_{k}(t)=v\left(t+\tilde{s}_{k}\right), t \geqslant 0$, is a Cauchy sequence in $L_{2}(\tilde{\Omega}, \tilde{P})$, uniformly in $t \geqslant 0$; i.e., for any $\varepsilon>0$ there is $l=l(\varepsilon)$ such that

$$
\sup _{t \geqslant 0} \tilde{E}\left|\tilde{v}_{k}-v_{l}(t)\right|^{2} \leq \varepsilon \quad \text { for all } \quad k \geqslant l(\varepsilon) .
$$

From (23) it follows that $\left\{P\left(t+s_{k}, d x\right)\right\}_{k \geqslant 1}$ is weakly compact uniformly with respect to $t \geqslant 0$ and denote by $\Pi(t, d x)$ a limit of it.

Thus we have obtained that $\widetilde{P}\left(t+h_{k}, d x\right), k \geqslant 1$, is weakly compact, uniformly with respect to $t \geqslant 0$ and the proof is complete.

ProOF OF TheOREM 1. Let $\left\{t_{k}^{\prime}\right\} \uparrow \infty$. Since $f_{i}, i=0,1, \cdots, m$, are asymptotically almost periodic in the variable $t \geqslant 0$, it follows that there exists a subsequence $\left\{t_{k}\right\} \uparrow \infty$ such that $f_{i}^{k}(t, x)=f_{i}\left(t+t_{k}, x\right)$ is Cauchy uniformly in $t \geqslant 0$ and $x$ in compact sets in $R^{n}$.

By hypotheses, the equation (1) is totally stable and Lemma 3 applies.

We get, for each $k \geqslant 1$, an equivalent differential equation $\left(1_{t_{k}}\right)$ whose solution $x_{k}(t)$, $t \geqslant 0$, generates the same probabilities $\tilde{P}\left(t+t_{k}, d x\right), t \geqslant 0$, as $\tilde{x}\left(t+t_{k}\right)$ and $\left\{x_{k}(t)\right\}_{k} \geqslant 1$ converges to a limit $x(t)$ in $L_{2}(\tilde{\Omega}, \tilde{P})$ uniformly in $t \geqslant 0$. Therefore $\left\{\widetilde{P}\left(t+t_{k}, d x\right)\right\}_{k} \geqslant 1$ is weakly convergent to a limit $P(t, d x) \in \mathscr{P}$ uniformly in $t \geqslant 0$ and the proof is complete.

RemarK 4. As is pointed out in Theorem 1, for any $L_{p}$-bounded solution $(p>2)$ $\hat{x}(t), t \geqslant 0$, in (1) we get the following convergence $\lim _{k \rightarrow \infty} \int_{R^{n}} \varphi(x) \hat{P}\left(t+t_{k}, d x\right)=$ $\int_{R^{n}} \varphi(x) P(t, d x)$ uniformly in $t \geqslant 0$ for each $\varphi \in C_{b}\left(R^{n}\right), \varphi$ Lipschitz continuous, for some sequence $\left\{t_{k}\right\} \uparrow \infty$.

Using Lemma 3 it is possible to extend this convergence to any $\varphi \in C^{1}\left(R^{n}\right)$, which 
fulfils

$$
\left|\frac{\partial \varphi}{\partial x}(x)\right|^{2} \leq k\left(1+|x|^{q}\right) \quad \text { with } \quad q \leq p .
$$

It gives the possibility to obtain asymptotic almost periodic property using the definition based on the moments of the bounded solution.

\section{REFERENCES}

[1] T. Yoshizawa, Stability Theory and the Existence of Periodic Solutions and Almost Periodic Solutions, Springer-Verlag, Applied Mathematical Sciences 14, 1975.

[2] Y. Hino AND T. YoshizAwa, Total Stability Property in Limiting Equations for a Functional Differential Eauation with Infinite Delay, Casopis pre pestovani matematiky, roc 111 (1986) Praha.

[3] C. Corduneanu, Almost Periodic Functions, Interscience Publishers, Interscience Tracts in Pure and Applied Mathematics, Nr. 22, 1968.

[4] N. IKeda AND S. Watanabe, Stochastic Differential Equations and Diffusion Processes, NorthHolland, 1981.

DEPARTMENT OF MATHEMATICS

INCREST

BD. PACCI 220, 79622 BuCHAREST

ROMANIA 